\title{
Calculation of the hyperfine structure transition energy and lifetime in the one-electron $\mathrm{Bi}^{82+}$ ion
}

\author{
M. Finkbeiner, B. Fricke \\ Fachbereich Physik, Universität Kassel, W-3500 Kassel, Germany \\ and \\ T. Kühl \\ GSI, Plankstrasse 1, Postfach 110552, W-6100 Darmstadt, Germany
}

Received 26 January 1993; revised manuscript received 10 March 1993; accepted for publication 10 March 1993

Communicated by J.P. Vigier

\begin{abstract}
We calculate the energy and lifetime of the ground state hyperfine structure transition in one-electron $\mathrm{Bi}^{82+}$. The influence of various distributions of the magnetic moment and the electric charge in the nucleus ${ }_{83}^{209} \mathrm{Bi}$ on energy and lifetime is studied.
\end{abstract}

One-electron atoms are the simplest real quantum mechanical systems one can think of. The first excited states of all these atoms are the hyperfine structure splittings of the $1 s_{1 / 2}$ ground state electron coupled to the nuclear spin. Indeed, going into detail these levels are very hard to calculate. In addition to the two-body kinematics relativistic and QED effects come into effect as well as all the influences arising from the internal structure of the nucleus; these are the extended electric as well as magnetic moments (Bohr-Weisskopf effect [1] ). In the case of a really heavy nucleus it is still not possible to calculate these effects in great detail.

Up to now the hyperfine structure transition of the hydrogen atom at $21 \mathrm{~cm}$ wavelength is the only one measured experimentally. Since heavy nuclei with only one electron are available in storage rings like the ESR at the GSI in Darmstadt, it is not only interesting but also possible to detect these transitions. In order to give the transition energy range where to look at, we discuss here the hyperfine structure energy and lifetime of the one-electron atom $\mathrm{Bi}^{82+}$ as example. The dominant part of the transition energy is given by the interaction of the magnetic moment of the electron with the nuclear magnetic moment. The effect which is due to the extension of the magnetic moment is called the Bohr-Weisskopf effect [1]. The other part (probably of the order of $1 \%$ of the magnetic moment part) is the QED contribution which will be calculated by another group [2]. The only calculation so far [3] is good only for small $Z$.

The transition energy between the hyperfine structure levels $i$ and $f$ is given as

$\Delta E=\int \Psi_{\mathrm{i}}^{*} c e \alpha \cdot A \Psi_{\mathrm{i}} \mathrm{d}^{3} r-\int \Psi_{\mathrm{f}}^{*} c e \alpha \cdot A \Psi_{\mathrm{f}} \mathrm{d}^{3} r$.

In the semiclassical approximation $\Psi_{\mathrm{i}}$ and $\Psi_{\mathrm{f}}$ are the wavefunctions of the $1 \mathrm{~s}_{1 / 2}$ electron in its $m_{j}$ substates $+\frac{1}{2}$ and $-\frac{1}{2} . \boldsymbol{A}$ is the vector potential of the nuclear magnetism. Using the four-component electronic wavefunction with $g(r)$ and $f(r)$, the large and small radial components, one gets

$\Delta E=-\int \frac{c e f(r) g(r)}{\pi} \frac{1}{r}[A(r) \times r]_{z} \mathrm{~d}^{3} r$. 
For a point-like magnetic moment with

$A(\boldsymbol{r})=\frac{\mu_{0}}{4 \pi} \frac{\boldsymbol{m} \times \boldsymbol{r}}{r^{3}}$

expression (2) becomes

$\Delta E=-\int \frac{c e f(r) g(r)}{\pi} \frac{1}{r} \frac{\mu_{0}}{4 \pi} \frac{m}{r}\left[\cos ^{2}(\theta)-1\right] \mathrm{d}^{3} r$.

After angular integration one gets

$\Delta E=+\frac{\mu_{0}}{4 \pi} \frac{8}{3} \operatorname{cem} \int_{0}^{\infty} f(r) g(r) \mathrm{d} r$.

If one now assumes a radially symmetric magnetic moment density function $\boldsymbol{M}(R)=\boldsymbol{m} w(R)$ and calculates eq. (2) one obtains the expression

$\Delta E=\frac{\mu_{0}}{4 \pi} \frac{8}{3} m c e \int_{0}^{\infty} f(r) g(r) \int_{0}^{r} w(R) \times 4 \pi R^{2} \mathrm{~d} R \mathrm{~d} r$.

There are two extreme cases, the magnetic moment distribution is given as a radial shell at $R_{0}$ and the splitting becomes

$\Delta E=\frac{\mu_{0}}{4 \pi} \frac{8}{3} m c e \int_{R_{0}}^{\infty} f(r) g(r) \mathrm{d} r$,

whereas the point dipole expression is given by eq. (4). Results (1)-(6) were obtained in ref. [1].

In the case of a fully quantum mechanical treatment $\boldsymbol{m}$ has to be replaced by $g_{\mathrm{N}} \mu_{\mathrm{N}} I$ with $I$ the nuclear spin operator, $\mu_{\mathrm{N}}$ the nuclear magneton and $g_{\mathrm{N}}$ the gyromagnetic ratio of the nucleus [4]. The wavefunction in (1) is then the fully coupled wavefunction made of the nuclear as well as the electronic part coupled to the good total angular momentum $F$. The final result for the interaction energy of a state with the quantum numbers $F$ and $m_{F}$ is

$$
\begin{aligned}
& \Delta E_{F m_{F}}=\frac{\mu_{0}}{4 \pi} g_{\mathrm{N}} \mu_{\mathrm{N}} c e \hbar \times \frac{4}{3} \int_{0}^{\infty} f(r) g(r) \mathrm{d} r\left[\left\langle\frac{1}{2}-\frac{1}{2} I m_{F}+\frac{1}{2} \mid F m_{F}\right\rangle^{2}\left(m_{F}+\frac{1}{2}\right)\right. \\
& -2\left\langle\frac{1}{2}-\frac{1}{2} I m_{F}+\frac{1}{2} \mid F m_{F}\right\rangle\left\langle\frac{1}{2}+\frac{1}{2} I m_{F}-\frac{1}{2} \mid F m_{F}\right\rangle \sqrt{I(I+1)-\left(m_{F}+\frac{1}{2}\right)\left(m_{F}-\frac{1}{2}\right)} \\
& \left.-\left\langle\frac{1}{2}+\frac{1}{2} I m_{F}-\frac{1}{2} \mid F m_{F}\right\rangle^{2}\left(m_{F}-\frac{1}{2}\right)\right],
\end{aligned}
$$

which is independent of $m_{F}$ as it should be. If we take up the special case $\mathrm{Bi}^{82+}$ with $I=\frac{9}{2}$ and $g_{\mathrm{N}}=4.1106 / I$ as measured [5-8] we get as the transition energy between the two states $F=5$ and $F=4$

$\Delta E=\frac{\mu_{0}}{4 \pi} \frac{4.1106}{I} \mu_{\mathrm{N}} \hbar c e \times \frac{4}{3} \times 10 \int_{0}^{\infty} f(r) g(r) \mathrm{d} r$.

Again if we assume a radially symmetric magnetic moment distribution the last integral has to be changed into $\int_{0}^{\infty} f(r) g(r) \int_{0}^{r} w(R) \times 4 \pi R^{2} \mathrm{~d} R \mathrm{~d} r$.

The radial functions $f(r)$ and $g(r)$ are determined from a numerical integration of the Dirac equation for the 
1s state in the most realistic electric potential arising from a two-parameter nuclear charge distribution,

$p(r)=\frac{\rho_{0}}{1+\exp \{[(r-c) / t] \times 4 \ln 3\}}$,

with $c=6.73 \pm 0.08 \mathrm{fm}$ and $t=2.12 \pm 0.16 \mathrm{fm}$ for ${ }^{209} \mathrm{Bi}$ [9] using the MCDF program of Desclaux [10]. Figure 1 shows the resulting $f(r) g(r)$ from the 1 s wavefunction for a point nucleus and an extended nucleus according to eq. (9). For the extension of the nuclear magnetic moment we use the model

$$
\begin{aligned}
w(R) & =k_{n} R^{n}, & & R \leqslant R_{0}, \\
& =0, & & R>R_{0},
\end{aligned}
$$

with the remaining exponent $n$. Assuming $R_{0}=c$ the value $k_{n}$ can be determined by the normalisation condition $\int_{0}^{c} k_{n} R^{n} \times 4 \pi R^{2} \mathrm{~d} R=k^{n} \times 4 \pi \frac{c^{n+3}}{n+3}=1$.

This parameter $n$ allows one to choose from a homogeneous magnetic moment distribution $(n=0)$ up to a distribution located at the surface of the nucleus $(n=\infty)$. The outermost proton in ${ }^{209} \mathrm{Bi}$ above the double magic nucleus ${ }^{208} \mathrm{~Pb}$ has an orbital moment $l=5$ which couples with the proton spin to become $I=\frac{9}{2}$. Due to the large orbital moment and the large deviation of $\mu=4.11 \mu_{\mathrm{N}}$ from the Schmidt value $\mu=2.624 \mu_{\mathrm{N}}$ we have chosen the parameter $n=2$ in our model, which corresponds to a magnetic moment distribution which is located fairly well in the outside region of the nucleus. Table 1 shows the resulting transition wavelength for various assumptions of the electric and magnetic distributions. Thus the most realistic value for the transition wavelength to be observed (without any QED correction, which may be of the order of $1 \%$ ) will be between 244.3 and $244.8 \mathrm{~nm}$. Of course this result is achieved with a "measured" $g$ value of 4.1106 . Unfortunately there are different measurements which yield values of 4.20(6) [11] and 4.25(14) [12]. A critical analysis nevertheless shows that the value 4.1106 due to ref. [8] probably is the most reliable one. But even this value is not the actual measured value because it has been corrected by the diamagnetic shielding factor of 1.0177 . In this context we have done accurate MCDF calculations of Bi using ref. [10] and determined a somewhat

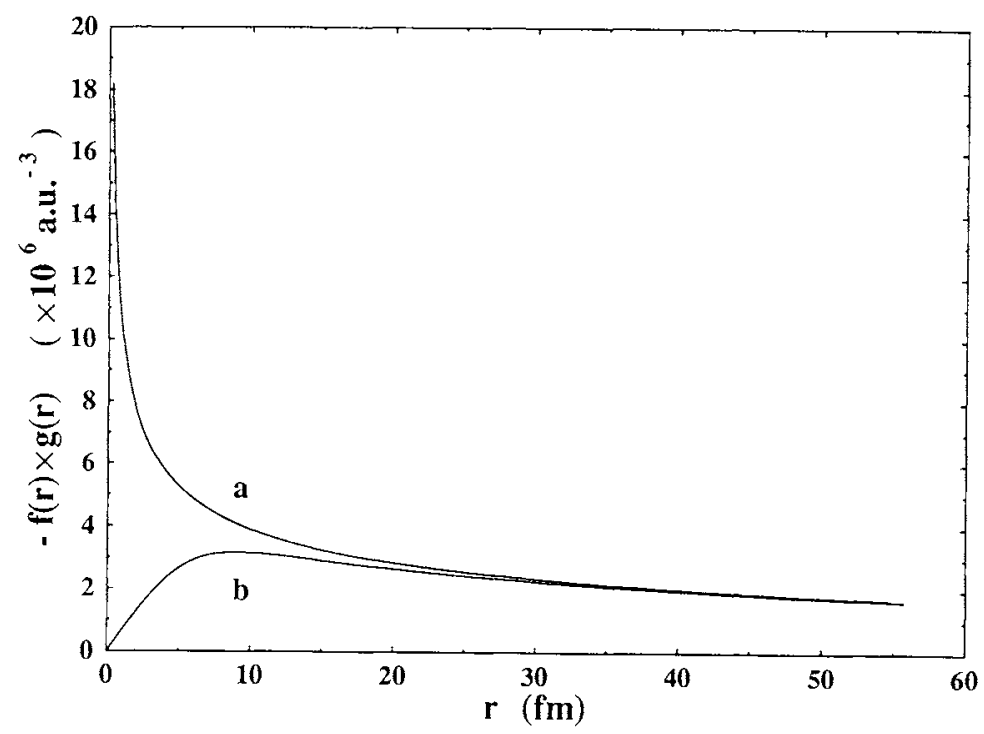

Fig. 1. The function $f(r) g(r)$ of the is relativistic wavefunction of ${ }^{209} \mathrm{Bi}^{82+}$ for a point nucleus (a) and an extended nucleus (b). 
Table 1

Transition wavelength for the ground state hyperfine transition in ${ }^{209} \mathrm{Bi}^{82+}$ in $\mathrm{nm}$ for various electric charge and magnetic moment distributions. The values where either the magnetic or the electric distribution in point-like are only of academic interest.

\begin{tabular}{lllll}
\hline $\begin{array}{l}\text { Magnetic } \\
\text { distribution }\end{array}$ & \multicolumn{3}{l}{ Electric charge distribution (eq. (9)) } \\
\cline { 2 - 5 } (eq. (10)) & point & $\begin{array}{l}c=6.81 \\
t=2.28\end{array}$ & $\begin{array}{l}c=6.73 \\
t=2.12\end{array}$ & $\begin{array}{l}c=6.65 \\
t=2.28\end{array}$ \\
\hline point & & 239.1 & 238.8 & 238.7 \\
$n=0$ & 212.7 & 243.8 & 243.5 & 243.3 \\
$n=2$ & 234.6 & 244.7 & 244.3 & 244.2 \\
$n=4$ & 236.4 & 245.1 & 244.8 & 244.6 \\
$n=\infty$ & 237.2 & 246.7 & 246.3 & 246.1 \\
\hline
\end{tabular}

Table 2

Lifetime for the ground state hyperfine transition in ${ }^{209} \mathrm{Bi}^{82+}$ in $\mu$ s for various wavelengths and electric charge distributions.

\begin{tabular}{lllll}
\hline $\begin{array}{l}\text { Wavelength } \\
(\mathrm{nm})\end{array}$ & \multicolumn{4}{l}{ Electric charge distribution (eq. (9)) } \\
\cline { 2 - 5 } & point & $\begin{array}{l}c=6.81 \\
t=2.28\end{array}$ & $\begin{array}{l}c=6.73 \\
t=2.12\end{array}$ & $\begin{array}{l}c=6.65 \\
t=2.28\end{array}$ \\
\hline 238 & & & 396.7 & 396.7 \\
240 & 396.9 & 396.7 & 406.8 & 406.8 \\
242 & 407.0 & 406.8 & 417.0 & 417.0 \\
244 & 417.3 & 417.0 & 427.5 & 427.5 \\
246 & 427.7 & 427.5 & 438.1 & 438.1 \\
248 & 438.3 & 438.0 & 448.8 & 448.8 \\
\hline
\end{tabular}

different diamagnetic shielding factor of 1.012. If this factor is used the transition wavelength would be between 242.9 and $243.4 \mathrm{~nm}$, which again is a $0.5 \%$ change.

Now we will calculate the lifetime for the hyperfine structure transition. In first order time dependent perturbation theory the transition rate is given by [13]

$w=\frac{2 \pi}{\hbar}\left|\left\langle\mathrm{f}\left|H^{\prime}\right| \mathrm{i}\right\rangle\right|^{2} \rho\left(E_{\mathrm{f}}\right)$

where $|i\rangle$ and $|f\rangle$ are again the fully coupled wavefunctions for the initial and final state, consisting of an electronic and a nuclear part. If we restrict ourselves to magnetic dipole transitions the interaction Hamiltonian is given by

$H^{\prime}=\sum_{M=-1}^{1} \boldsymbol{J} \cdot \boldsymbol{A}_{1 M}$

where $J=-e \alpha$ is the current density of the ion and $A_{1 M}$ is the vector potential of an external field for the magnetic dipole [13],

$A_{L M}=-\eta j_{L}(k r) \sum_{\nu=-1}^{1}\langle 1 \nu 1 M-\nu \mid 1 M\rangle Y_{1 M-\nu} \xi_{\nu}$.

Using $\eta^{2}=4 \pi \hbar \omega / R$ and $\rho\left(E_{\mathrm{f}}\right)=R / \pi \hbar c^{\prime}[13]$ the angular integration in eq. (11) can be carried out analytically. The rate for transitions from $F=I+\frac{1}{2}, m_{F}$ to $F=I-\frac{1}{2}, m_{F}^{\prime}$ is finally given in SI units as

$$
\begin{aligned}
& w=\frac{32}{3} \omega \alpha\left(\int_{0}^{\infty} j_{1}(k r) f(r) g(r) r^{2} \mathrm{~d} r\right)^{2} \\
& \times\left[\delta_{m_{F}^{\prime}+1 / 2 m_{F}+1 / 2}\left\langle\frac{1}{2}-\frac{1}{2} I m_{F}^{\prime}+\frac{1}{2} \mid I-\frac{1}{2} m_{F}^{\prime}\right\rangle\left\langle\frac{1}{2}-\frac{1}{2} I m_{F}+\frac{1}{2} \mid I+\frac{1}{2} m_{F}\right\rangle(-1 / \sqrt{2})\right.
\end{aligned}
$$

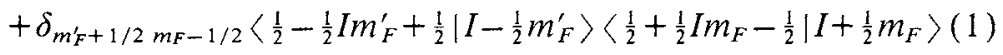

$$
\begin{aligned}
& +\delta_{m_{F}^{\prime}-1 / 2 m_{F}+1 / 2}\left\langle\frac{1}{2}+\frac{1}{2} I m_{F}^{\prime}-\frac{1}{2} \mid I-\frac{1}{2} m_{F}^{\prime}\right\rangle\left\langle\frac{1}{2}-\frac{1}{2} I m_{F}+\frac{1}{2} \mid I+\frac{1}{2} m_{F}\right\rangle(-1) \\
& \left.+\delta_{m_{F}^{\prime}-1 / 2 m_{F-1 / 2}}\left\langle\frac{1}{2}+\frac{1}{2} I m_{F}^{\prime}-\frac{1}{2} \mid I-\frac{1}{2} m_{F}^{\prime}\right\rangle\left\langle\frac{1}{2}+\frac{1}{2} I m_{F}-\frac{1}{2} \mid I+\frac{1}{2} m_{F}\right\rangle(1 / \sqrt{2})\right]^{2} \text {. }
\end{aligned}
$$

One finds that the sum of the three transition rates from $F=I+\frac{1}{2}, m_{F}$ to $F=I-\frac{1}{2}, m_{F}^{\prime}$ with $m_{F}^{\prime}=m_{F}-1$, $m_{F}^{\prime}=m_{F}$, and $m_{F}^{\prime}=m_{F}+1$ is independent of $m_{F}$ as it should be. In the case of $\mathrm{Bi}^{82+}$ with $I=\frac{9}{2}$ this sum becomes 
$w_{\mathrm{tot}}=\frac{32}{3} \omega \alpha \times \frac{9}{10}\left(\int_{0}^{\infty} j_{1}(k r) f(r) g(r) r^{2} \mathrm{~d} r\right)^{2}$

and the lifetime is $\tau=1 / w_{\text {tot. }}$. Table 2 shows the lifetime for various wavelengths and electric distributions. Since in (12) the current density of the nucleus is neglected, the resulting lifetime does not explicitly depend on the distribution of nuclear magnetism. The only effect of this distribution is that it influences the transition energy and thus the angular frequency $\omega$ and wavenumber $k$ appearing in (13). However, table 2 shows that for fixed wavelength, variations of the electric distribution affecting the radial functions $f(r)$ and $g(r)$ in (13) have only a minor effect on the lifetime too. Thus the main influence of the electric distribution of the nucleus on the lifetime is that it affects the transition energy according to eqs. (7) and (8) which are given in table 1.

This discussion shows that the uncertainty in the transition energy and lifetime due to the uncertainty in the electric charge and magnetic moment distribution still is of the order of $1 \%-2 \%$. Even if an accurate value of the QED contribution is available this uncertainty would not be much smaller.

Nevertheless the measurement of this transition is very important, first because this transition has up to now only been measured in $\mathrm{H}$, and second because a systematic study for many transitions in the region of large $Z$ probably will lead to a better understanding of the dominant uncertainty which results from the magnetic moment distribution.

One of us (M.F.) would like to thank the Deutsche Forschungsgemeinschaft for financial support. The use of the GSI computer is also gratefully acknowledged.

\section{References}

[1] A. Bohr and V.F. Weisskopf; Phys. Rev. 77 (1950) 94.

[2] G. Soff, private communication.

[3] S.J. Brodsky and G.W. Erickson, Phys. Rev. 148 (1966) 26.

[4] E.M. Rose, Relativistische Elektronentheorie (Bibliographisches Institut, Mannheim, 1961 ).

[5] Y. Ting and D. Williams, Phys. Rev. 89 (1953) 595.

[6] C.M. Lederer and V.S. Shirley, Table of isotopes, 7th Ed. (Wiley, New York, 1978).

[7] F.D. Feiock and W.R. Johnson, Phys. Rev. 187 (1969) 39.

[8] P. Raghavan, At. Data Nucl. Data Tables 42 (1989) 189.

[9] Landolt-Börnstein, Zahlenwerte and Funktionen aus Naturwissenschaft und Technik, Gruppe I, Bd. 2. Kernradien (1967) p. 36.

[10] J.P. Desclaux, Comput. Phys. Commun. 9 (1975) 31.

[1 1 ] A.M. Crooker and G. Shipley, Can. J. Phys. 48 (1970) 730.

[12] D.A. Landman and A. Lurio, Phys. Rev. A 1 (1970) 1330.

[13] H.R. Rosner and C.P. Bhalla, Z. Phys. 231 (1956) 347. 